\title{
Лазерная генерация многопериодных квантово-каскадных лазеров на длине волны излучения 8 мкм при комнатной температуре
}

\author{
(C) А.В. Бабичев ${ }^{1,2}$, А.Г. Гладышев ${ }^{2}$, А.С. Курочкин ${ }^{1}$, Е.С. Колодезный ${ }^{1}$, Г.С. Соколовский ${ }^{1,3,4}$, В.Е. Бугров ${ }^{1}$, \\ Л.Я. Карачинский ${ }^{1,2,3}$, И.И. Новиков ${ }^{1,2,3}$, А. Bousseksou ${ }^{5}$, А.Ю. Егоров ${ }^{1, \uparrow}$ \\ ${ }^{1}$ Санкт-Петербургский национальный исследовательский университет информационных технологий, механики и оптики, \\ 197101 Санкт-Петербург, Россия \\ 2 „Коннектор Оптикс“, \\ 194292 Санкт-Петербург, Россия \\ ${ }^{3}$ Физико-технический институт им. А.Ф. Иоффе Российской академии наук, \\ 194021 Санкт-Петербург, Россия \\ ${ }^{4}$ Санкт-Петербургский государственный электротехнический университет „ЛЭТИ“ им. В.И. Ульянова (Ленина), \\ 197376 Санкт-Петербург, Россия \\ ${ }^{5}$ Center of Nanoscience and Nanotechnology (C2N), Université, Paris Sud and Paris-Saclay, \\ 91405 Orsay cedex, France \\ 『 E-mail: anton.egorov@connector-optics.com
}

(Получена 1 фревраля 2018 г. Принята к печати 12 фревраля 2018 г.)

\begin{abstract}
Продемонстрирована лазерная генерация многопериодных квантово-каскадных лазеров при комнатной температуре на длине волны излучения 8 мкм при накачке импульсами тока. Гетероструктура квантовокаскадного лазера на основе гетеропары твердых растворов $\mathrm{In}_{0.53} \mathrm{Ga}_{0.47} \mathrm{As} / \mathrm{Al}_{0.48} \mathrm{In}_{0.52} \mathrm{As}$, согласованной с подложкой InP, выращена методом молекулярно-пучковой эпитаксии и состоит из 50 идентичных каскадов, помещенных в волновод с верхней воздушной обкладкой. Плотность порогового тока при температуре $300 \mathrm{~K}$ для полоскового лазера с длиной резонатора 1.4 мм и шириной полоска 24 мкм составляет $\sim 5.1$ кА/см².
\end{abstract}

DOI: $10.21883 /$ FTP.2018.08.46226.8834

\section{1. Введение}

Квантово-каскадные лазеры (ККЛ), излучающие в спектральном диапазоне 7-8 мкм, могут эффективно применяться для медицинских применений и проведения дистанционного газоанализа. В частности, лазеры с длиной волны 7.3-7.9 мкм применяются для детектирования $\mathrm{SO}_{x}$ [1], метана [2], $\mathrm{H}_{2} \mathrm{~S}, \mathrm{C}_{2} \mathrm{H}_{2}, \mathrm{~N}_{2} \mathrm{O}$ [3], тринитротолуола [4].

$\mathrm{SO}_{2}$ входит в список наиболее распространенных примесей, присутствующих в газовых магистралях медицинских учреждений. В силу токсичности $\mathrm{SO}_{2}$ особое внимание обращено к созданию компактных систем детектирования оксида серы, непосредственно расположенных в палатах пациентов. Данные системы основаны на применении ККЛ [5].

В свою очередь детектирование метана (на длине волны излучения 7.8 мкм) при помощи ККЛ может применяться для удаленного контроля утечек на газопроводах и химических производствах. Более того, присутствие $\mathrm{CH}_{4}$ в выдыхаемом человеком воздухе свидетельствует о ряде заболеваний желудочно-кишечного тракта (синдром раздраженного кишечника, рак толстой кишки, затруднение дефекации $[6,7])$. Помимо технологии газоанализа выдыхаемого воздуха, также представляет интерес детектирование различных жидкостей при осаждении на поверхность ККЛ (геометрия ККЛ с воздушной верхней обкладкой [8]).

К настоящему моменту в литературе представлен ряд принципиальных схем активной области ККЛ спектрального диапазона 7-8 мкм: на основе двухфононного $[1,9,10]$, трехфононного резонансного рассеяния электронов [11], на основе конструкций со сверхрешетками $[12,13]$, с переходами „связанное состояние-непрерывный спектр“ $[14,15]$, с нерезонансным выбросом носителей заряда [16,17], с выбросом носителей заряда за счет однофононного рассеяния в континуум $[18,19]$, с двух-уровневым верхним состоянием [20].

Основными технологиями выращивания гетероструктур ККЛ являются молекулярно-пучковая эпитаксия (МПЭ) и металлоорганическая газофазная эпитаксия (МОГФЭ). МПЭ позволяет реализовать лазеры с более высокой квантовой эффективностью [21]. Многопериодная геометрия активной области гетероструктур ККЛ [22-24] также положительно сказывается на величине квантовой эффективности. В свою очередь изготовление данного типа гетероструктур требует поддержания высокой идентичности химического состава и толщин слоев, формирующих каскады, на протяжении всего эпитаксиального процесса [25,26]. Использование промышленных установок МПЭ удовлетворяет этим требованиям [27,28] и положительно отражается на структурном качестве выращенных гетероструктур.

В настоящей работе представлены результаты по формированию и исследованию оптических характеристик многопериодных ККЛ, излучающих в импульсном режиме при комнатной температуре на длине волны 8 мкм.

\section{2. Эксперимент}

Гетероструктура ККЛ выращена компанией „Коннектор Оптикс“ с использованием промышленной установки МПЭ Riber 49, оснащенной твердотельным источником мышьяка крекерного типа и источниками 


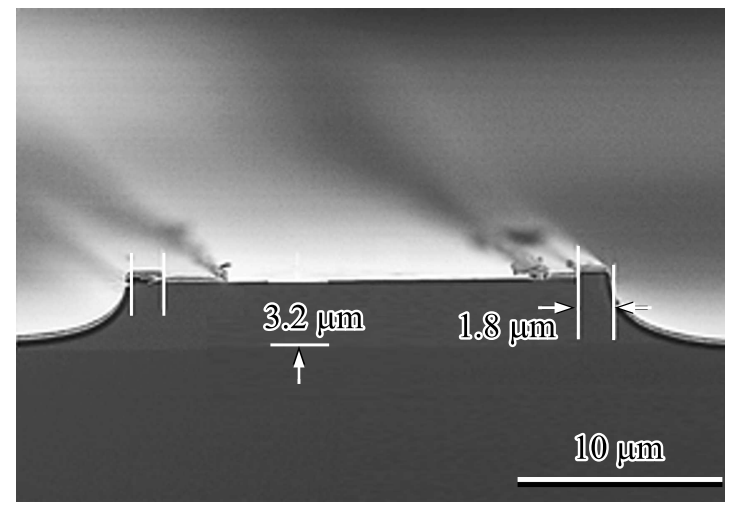

Рис. 1. Изображение скола изготовленного ККЛ, полученное методом сканирующей электронной микроскопии.

марки ABI 1000 для создания потоков галлия и индия. Подложка InP с ориентацией (001) легирована серой до уровня $3 \cdot 10^{18} \mathrm{~cm}^{-3}$. Нижняя обкладка волновода сформирована на основе последовательно выращенных на подложке слоев InP (толщиной 2 мкм) и $\operatorname{In}_{0.53} \mathrm{Ga}_{0.47} \mathrm{As}$ (толщиной 0.5 мкм). Активная область, включающая 50 квантовых каскадов, сформирована на основе механически-ненапряженных квантовых ям состава $\operatorname{In}_{0.53} \mathrm{Ga}_{0.47} \mathrm{As}$ с барьерными слоями $\mathrm{Al}_{0.48} \mathrm{In}_{0.52} \mathrm{As}$. Для построения каскада использована схема с двухфононным резонансным рассеянием носителей заряда. После выращивания каскадов последовательно формировались слои $\operatorname{In}_{0.53} \mathrm{Ga}_{0.47} \mathrm{As}$ с уровнем легирования $1 \cdot 10^{17}$ и $1 \cdot 10^{19} \mathrm{~cm}^{-3}$, толщинами 0.1 и 0.02 мкм соответственно. Детальное описание конструкции гетероструктуры ККЛ представлено в работе [29].

При формировании кристалла ККЛ использована геометрия волновода с верхней воздушной обкладкой [9]. Формирование полоска лазера начиналось с вытравливания глубокой мезы жидкостным травлением (на основе раствора $\left.\mathrm{HBr}: \mathrm{HNO}_{3}: \mathrm{H}_{2} \mathrm{O}\right)$. В качестве маски для травления полоска использовался слой оксида кремния $\left(\mathrm{SiO}_{2}\right)$, локально вытравленный во фторсодержащей плазме под маской фоторезиста AZ5214. После формирования профиля мезы и удаления маски на основе $\mathrm{SiO}_{2}$ производилась пассивация боковых стенок мезы путем напыления слоя нитрида кремния $\left(\mathrm{Si}_{3} \mathrm{~N}_{4}\right)$ методом плазмохимического осаждения. Открытие окон в слое $\mathrm{Si}_{3} \mathrm{~N}_{4}$ под металлизацию проводилось путем реактивного ионного травления в смеси газов $\mathrm{CHF}_{3} / \mathrm{O}_{2}$ под маской из фоторезиста. Верхняя металлизация формировалась за счет высоковакуумного напыления (через маску фоторезиста AZ2070) последовательных слоев титана и золота с толщинами 20 и 200 нм соответственно. Перед напылением нижнего металлического контакта пластина утончалась до толщины 150 мкм. В качестве нижней металлизации использовалась последовательность слоев титан/золото с толщинами 20 и 200 нм соответственно. Зеркала лазеров формировались скалыванием. Ширина полоска вблизи поверхности гетероструктуры составила 24 мкм, длина резонатора 1.4 мм. Изображение скола изготовленного ККЛ, полученное методом сканирующей электронной микроскопии, представлено на рис. 1. Отражающее и антиотражающее покрытия на сколотые грани лазера не наносились. Монтаж производился на посеребренный медный теплоотвод.

Исследования смонтированных на теплоотвод экспериментальных образцов ККЛ проводились в температурном диапазоне 78-300 К. Вольт-амперные характеристики (BAX) измерялись при пропускании импульсов тока с длительностью 100 нс на частоте 84 кГц. Спектры стимулированного излучения ККЛ регистрировались с помощью Фурье-спектрометра и фотоприемника на основе $\mathrm{HgCdTe}$.

\section{3. Результаты и их обсуждение}

Типичные экспериментальные ВАХ, измеренные при различных температурах $(T)$, представлены на рис. 2. Изломы на вольт-амперных характеристиках связаны с конкурированием излучения нескольких мод [15]. Значение порогового напряжения $V_{\text {th }}[30]$ составляет 12.1 и 12.6 В при $T=78$ и $300 \mathrm{~K}$ соответственно. Минимальное значение $V_{\text {th }}=10.4 \mathrm{~B}$ соответствует температуре $250 \mathrm{~K}$. Падение напряжения при переходе от активной области каскада в область инжектора следующего каскада $E_{\text {def }} / q-$ „defect voltage“"определяется на основе выражения (1) для полного падения напряжения на гетероструктуре [31]:

$$
V=N h v+N E_{\mathrm{def}}+q V_{s},
$$

где $h v-$ энергия кванта, $q$ - заряд электрона, $V_{s}-$ паразитное падение напряжения на контактах и толстых слоях обкладок волновода, $N$ - число каскадов в гетероструктуре лазера. Точное определение $V_{s}$ требует выращивания гетероструктуры ККЛ без активной обла-

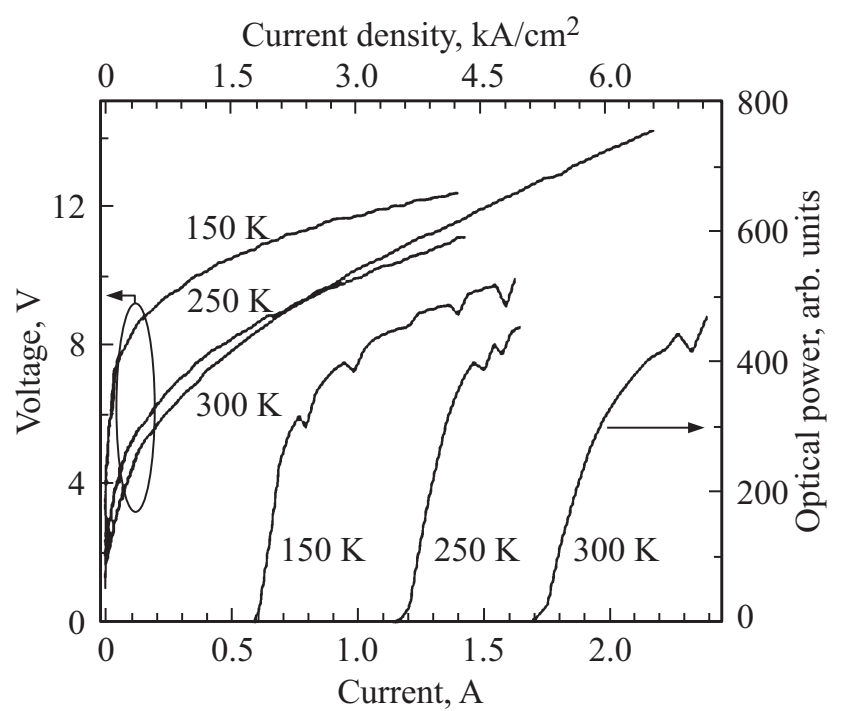

Рис. 2. Вольт-амперные характеристики, измеренные при различных температурах (левая ось $Y$ ). Ватт-амперные характеристики, измеренные при различных температурах (правая ось $Y)$. 


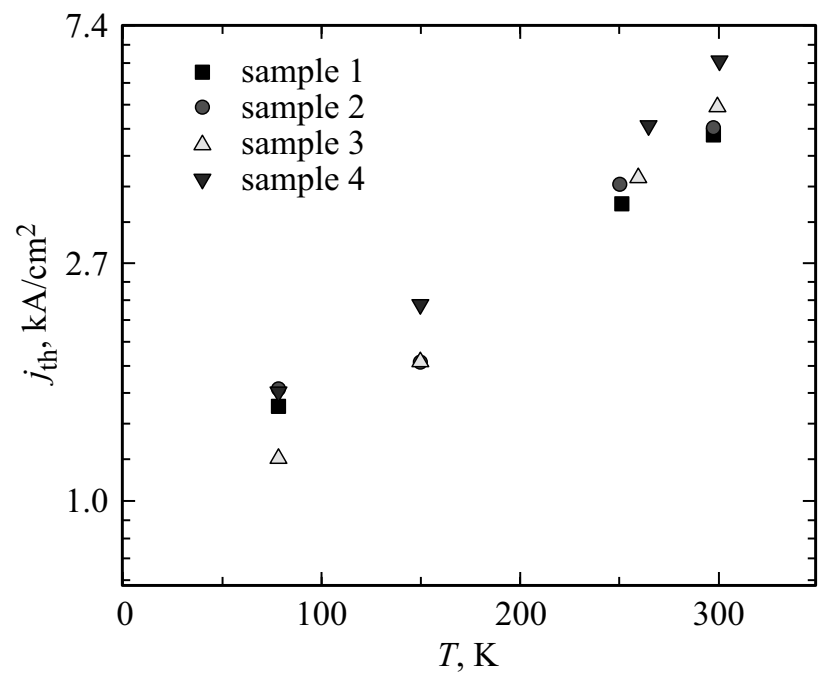

Pис. 3. Температурные зависимости плотности порогового тока ККЛ для 4 исследованных образцов ККЛ.

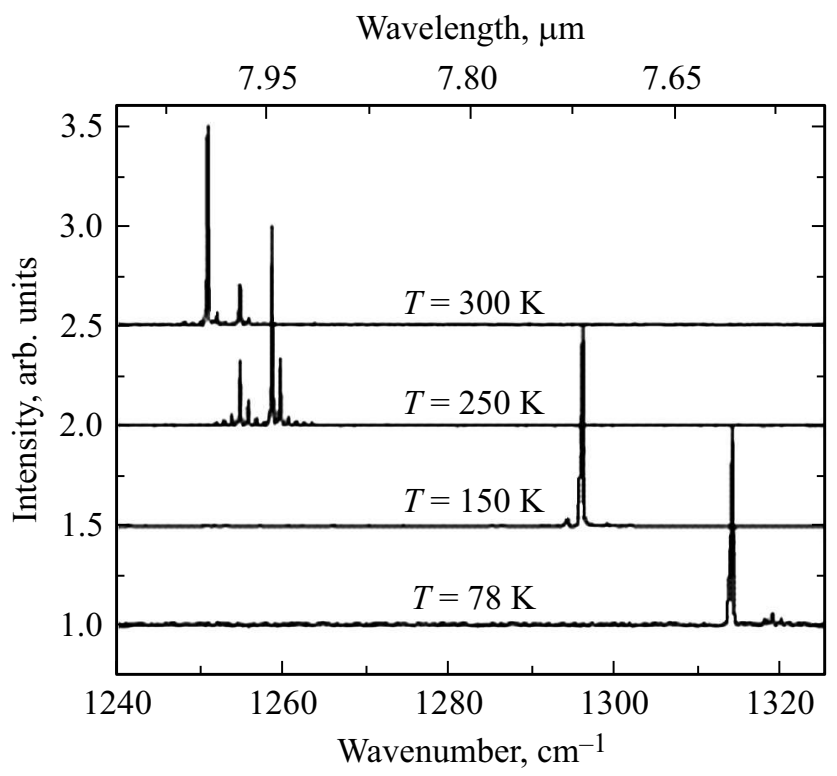

Рис. 4. Спектры стимулированного излучения образцов ККЛ, измеренные при различных температурах.

сти [31]. Для исключения недооценки величины $E_{\mathrm{def}}$ за счет вклада $V_{s}$, последней величиной в ряде случаев пренебрегают [32]. Полагая $V_{s}=0$, определенная величина $E_{\mathrm{def}}(T=78 \mathrm{~K})$ и $E_{\mathrm{def}}(T=300 \mathrm{~K})$ составляет 88 и 133 мэВ соответственно. $E_{\mathrm{def}}-$ разница в энергии между нижним лазерным уровнем и основным состоянием в области инжектора. С одной стороны, большая величина $E_{\text {def }}$ приводит к чрезмерному падению напряжения в структуре ККЛ и, как следствие, отрицательно сказывается на эффективности работы лазера [31]. С другой стороны, малые значения $E_{\text {def }}$ увеличивают паразитный вклад в кинетику носителей заряда за счет теплового заброса электронов из основного состояния инжектора на нижний уровень предыдущего каскада, уменьшая инверсию населенности лазера и, как следствие, пони- жая эффективность ККЛ. Типичная величина $E_{\mathrm{def}}$ для конструкции ККЛ с легированным инжектором составляет 130-160 мэВ [33-36], что хорошо соотносится с полученными результатами.

Зависимости пороговой плотности тока $\left(j_{\text {th }}\right)$ от температуры для исследованных гетероструктур представлены на рис. 3. При $T=300 \mathrm{~K}$ минимальная величина $j_{\text {th }}$ составляет $5.1 \kappa \mathrm{A} / \mathrm{cm}^{2}$. Аппроксимация экспериментальных зависимостей $j_{\text {th }}(T)$ на основе выражения $j_{\text {th }}(T)=j_{0} \exp \left(T / T_{0}\right)$ [37], дает значения характеристической температуры стабильности порогового тока $T_{0}$ в диапазоне $147-159 \mathrm{~K}$, плотности порогового тока при нулевой температуре $j_{0}=0.7-0.9 \kappa \mathrm{A} / \mathrm{cm}^{2}$.

На рис. 4 представлены спектры лазерной генерации, измеренные при различных температурах. Видно, что имеет место многомодовая лазерная генерация. С увеличением температуры наблюдается сдвиг максимума интенсивности спектра стимулированного излучения в длинноволновую область, обусловленный уменьшением разрыва зоны проводимости на гетерогранице с ростом температуры [32]. Максимум интенсивности, измеренный при $T=300 \mathrm{~K}$ соответствует длине волны 8 мкм. Межмодовое расстояние между продольными модами $\Delta \lambda$ составляет $\sim 0.0061$ мкм.

\section{4. Заключение}

В работе продемонстрирована лазерная генерация полосковых лазеров, изготовленных из многопериодной гетероструктуры квантово-каскадного лазе$\mathrm{pa}$, состоящей из 50 каскадов на основе гетеропары $\mathrm{In}_{0.53} \mathrm{Ga}_{0.47} \mathrm{As} / \mathrm{Al}_{0.48} \mathrm{In}_{0.52} \mathrm{As}$. Лазерная генерация наблюдается на длине волны излучения 8 мкм при температуре $300 \mathrm{~K}$ при накачке импульсами тока. Плотность порогового тока в полосковых лазерах с длиной резонатора $1.4 \mathrm{Mм}$ и шириной полоска 24 мкм составляет $j_{\text {th }} \sim 5.1 \mathrm{\kappa A} / \mathrm{cm}^{2}$. Данное значение сопоставимо с результатами, представленными ранее для аналогичной конструкции активной области гетероструктуры ККЛ, выращенной методом МОГФЭ. Полученные результаты свидетельствуют о необходимости дальнейшей оптимизации конструкции гетероструктуры ККЛ для улучшения рабочих характеристик лазера.

Работа выполнена при поддержке Министерства образования и науки РФ в рамках Федеральной целевой программы „Исследования и разработки по приоритетным направлениям развития научно-технологического комплекса России на 2014-2020 годы“, шифр 2016-14-579-0009, соглашение № 14.578.21.0204 от 3 октября 2016 г., уникальный идентификатор RFMEFI57816X0204.

\section{Список литературы}

[1] J.S. Yu, S. Slivken, M. Razeghi. Semicon Sci. Technol., 25, 125015 (2010.)

[2] A.P.M. Michel, J. Kapit, M.F. Witinski, R. Blanchard. Appl. Optics, 56 (11), E23 (2017). 
[3] L. Joly, V. Zéninari, T. Decarpenterie, J. Cousin, B. Grouiez, D. Mammez, G. Durry, M. Carras, X. Marcadet, B. Parvitte. Laser Phys., 21 (4), 805 (2011).

[4] J. Hildenbrand, J. Herbst, J. Wöllenstein, A. Lambrecht. Proc. SPIE ed. by M. Razeghi et al. (San Jose, CA, USA, 2009) v. 7222 , p. $72220 B$.

[5] H. Zimmermann, M. Wiese, L. Fiorani, A. Ragnoni. JSSS., 6 (1), 155 (2017).

[6] D. Kunkel, R.J. Basseri, M.D. Makhani, K. Chong, C. Chang, M. Pimentel. Dig. Dis. Sci., 56 (6), 1612 (2011).

[7] M. Jahjah, W. Ren, P. Stefański, R. Lewicki, J. Zhang, W. Jiang, J. Tarka, F.K. Tittel. Analyst, 139 (9), 2065 (2014).

[8] V. Moreau, R. Colombelli, R. Perahia, O. Painter, L.R. Wilson, A.B. Krysa. Opt. Express, 16 (9), 6387 (2008).

[9] A. Bousseksou, R. Colombelli, A. Babuty, Y. De Wilde, Y. Chassagneux, C. Sirtori, G. Patriarche, G. Beaudoin, I. Sagnes. Opt. Express, 17 (11), 9391 (2009).

[10] Y. Bidaux, R. Terazzi, A. Bismuto, T. Gresch, S. Blaser, A. Muller, J. Faist. J. Appl. Phys., 118 (9), 093101 (2015).

[11] R. Maulini, A. Lyakh, A. Tsekoun, C.K.N. Patel. Opt. Express, 19, 17203 (2011).

[12] C. Gmachl, A. Tredicucci, F. Capasso, A.L. Hutchinson, D.L. Sivco, J.N. Baillargeon, A.Y. Cho. Appl. Phys. Lett., 72, 3130 (1998).

[13] S. Slivken, A. Matlis, A. Rybaltowski, Z. Wu, M. Razeghi. Appl. Phys. Lett., 74 (19), 2758 (1999).

[14] R.P. Leavitt, J.L. Bradshaw, K.M. Lascola, G.P. Meissner, F. Micalizzi, F.J. Towner, J.T. Pham. Opt. Eng., 49, 111109 (2010).

[15] M. Troccoli, X. Wang, J. Fan. Opt. Eng., 49 (11), 111106 (2010).

[16] M. Troccoli, A. Lyakh, J. Fan, X. Wang, R. Maulini, A.G. Tsekoun, R. Go, C.K.N. Patel. Opt. Mater. Express, 3, 1546 (2013).

[17] R.M. Briggs, C. Frez, M. Fradet, S. Forouhar, R. Blanchard, L. Diehl, C. Pflügl. Opt. Express, 24 (13), 14589 (2016).

[18] K. Fujita, S. Furuta, A. Sugiyama, T. Ochiai, T. Edamura, N. Akikusa, M. Yamanishi, H. Kan. Appl. Phys. Lett., 91 (14), 141121 (2007).

[19] B. Schwarz, C.A. Wang, L. Missaggia, T.S. Mansuripur, P. Chevalier, M.K. Connors, D. McNulty, J. Cederberg, G. Strasser, F. Capasso. ACS Photonics 4 (5), 1225 (2017).

[20] K. Fujita, M. Yamanishi, S. Furuta, A. Sugiyama, T. Edamura. Appl. Phys. Lett., 101 (18), 181111 (2012).

[21] M. Troccoli. IEEE J. Select. Top. Quantum Electron, 21, 61 (2015).

[22] C. Gmachi, F. Capasso, A. Tredicucci, D.L. Sivxo, R. Kohler, A.L. Hutchinson, A.Y. Cho. IEEE J. Select. Top. Quant. Electron., 5 (3), 808 (1999).

[23] H. Li, S. Katz, A. Vizbaras, G. Boehm, M.C. Amann. IEEE Photonics Technol. Lett., 22 (24), 1811 (2010).

[24] M. Razeghi, S. Slivken. Proc. SPIE ed. by J. Rutkowski, A. Rogalski (2003) v. 5136, p. 317

[25] O. Fedosenko, M. Chashnikova, S. Machulik, J. Kischkat, M. Klinkmüller, A. Aleksandrova, G. Monastyrskyi, M.P. Semtsiv, W.T. Masselink. J. Cryst. Growth., 323, 484 (2011).

[26] H. Zhu, F. Wang, Q. Yan, C. Yu, J. Chen, G. Xu, L. He, L. Li, L. Chen, A.G. Davies, E.H. Linfield, J. Hao, P.B. Vigneron, R. Colombelli. Appl. Phys. Lett., 109 (23), 231105 (2016).

[27] M. Garcia, F.J. Vermersch, X. Marcadet, S. Bansropun, M. Carras, A. Wilk, C. Sirtori. Proc. SPIE (San Jose, CA, USA, 2006) v. 6133, p. 613304.
[28] A.V. Babichev, A. Bousseksou, N.A. Pikhtin, I.S. Tarasov, E.V. Nikitina, A.N. Sofronov, D.A. Firsov, L.E. Vorobjev, I.I. Novikov, L.Ya. Karachinsky, A.Yu. Egorov. Semiconductors, 50 (10), 1299 (2016).

[29] A.V. Babichev, A.G. Gladyshev, A.V. Filimonov, V.N. Nevedomskii, A.S. Kurochkin, E.S. Kolodeznyi, G.S. Sokolovskii, V.E. Bugrov, L.Ya. Karachinsky, I.I. Novikov, A. Bousseksou, A.Yu. Egorov. Tech. Phys. Lett., 43 (7), 666 (2017).

[30] M. Razeghi. IEEE J. Sel. Top. Quantum Electron., 15 (3), 941 (2009).

[31] M.D. Escarra, A.J. Hoffman, K.J. Franz, S.S. Howard, R. Cendejas, X. Wang, J.-Y. Fan, C. Gmachl. Appl. Phys. Lett., 94 (25), 251114 (2009).

[32] Y. Bai, S. Slivken, S. Kuboya, S.R. Darvish, M. Razeghi. Nat. Photonics, 4 (2), 99 (2010).

[33] A. Lyakh, R. Maulini, A. Tsekoun, R. Go, S. Von der Porten, C. Pflugl, L. Diehl, F. Capasso, C.K.N. Patel. Proc. Natl. Acad. Sci., 107 (44), 18799 (2010).

[34] A. Evans, S.R. Darvish, S. Slivken, J. Nguyen, Y. Bai, M. Razeghi. Appl. Phys. Lett., 91 (7), 071101 (2007).

[35] J. Faist. Appl. Phys. Lett., 90 (25), 253512 (2007).

[36] N. Bandyopadhyay, Y. Bai, S. Slivken, M. Razeghi. Appl. Phys. Lett., 105 (7), 071106 (2014).

[37] A.Y. Egorov, A.V. Babichev, L.Y. Karachinsky, I.I. Novikov, E.V. Nikitina, M. Tchernycheva, A.N. Sofronov, D.A. Firsov, L.E. Vorobjev, N.A. Pikhtin, I.S. Tarasov. Semiconductors, 49 (11), 1527 (2015).

Редактор А.Н. Смирнов

\section{Room temperature lasing of multi-stage quantum-cascade lasers at $8 \mu \mathrm{m}$ wavelength}

A.V. Babichev 1,2, A.G. Gladyshev ${ }^{2}$, A.S. Kurochkin ${ }^{1}$, E.S. Kolodeznyi ${ }^{1}$, G.S. Sokolovskii ${ }^{1,3,4}$, V.E. Bougrov ${ }^{1}$, L.Ya. Karachinsky ${ }^{1,2,3}$, I.I. Novikov ${ }^{1,2,3}$,

A.G. Bousseksou ${ }^{5}$, A.Yu. Egorov ${ }^{1}$

${ }^{1}$ ITMO University,

197101 St. Petersburg, Russia

${ }^{2}$ Connector Optics LLC,

194292 St. Petersburg, Russia

${ }^{3}$ loffe Institute, 194021 St. Petersburg, Russia

${ }^{4}$ St. Petersburg State Electrotechnical University „LETI", 197376 St. Petersburg, Russia

${ }^{5}$ Center of Nanoscience and Nanotechnology (C2N), Université Paris Sud and Paris-Saclay,

91405 Orsay cedex, France

Abstract Lasing of $8 \mu \mathrm{m}$ range multi-period quantum-cascade lasers at room temperature under pulsed pumping is demonstrated. The heterostructure of a quantum-cascade laser based on $\mathrm{In}_{0.53} \mathrm{Ga}_{0.47} \mathrm{As} / \mathrm{Al}_{0.48} \mathrm{In}_{0.52} \mathrm{As}$ heteropair coordinated with the $\mathrm{InP}$ substrate was grown by the molecular beam epitaxy technique and consists of 50 identical cascades placed in a waveguide with a top air cover. Stripe lasers with the cavity length of $1.4 \mathrm{~mm}$ and a stripe width of $24 \mu \mathrm{m}$ show the threshold current density of $\sim 5.1 \mathrm{kA} / \mathrm{cm}^{2}$ at a temperature of $300 \mathrm{~K}$. 